Review

\title{
Upconversion Nanoparticles for Photodynamic Therapy and Other Cancer Therapeutics
}

\author{
Chao Wang, Liang Cheng, Zhuang Liu ${ }^{凶}$ \\ Jiangsu Key Laboratory for Carbon-Based Functional Materials \& Devices, Institute of Functional Nano \& Soft Materials (FUNSOM), Soo- \\ chow University, Suzhou, Jiangsu 215123, China. \\ $\triangle$ Corresponding author: E-mail: zliu@suda.edu.cn. \\ ( ) Ivyspring International Publisher. This is an open-access article distributed under the terms of the Creative Commons License (http://creativecommons.org/ \\ licenses/by-nc-nd/3.0/). Reproduction is permitted for personal, noncommercial use, provided that the article is in whole, unmodified, and properly cited.
}

Received: 2012.09.24; Accepted: 2012.10.23; Published: 2013.03.25

\begin{abstract}
Photodynamic therapy (PDT) is a non-invasive treatment modality for a variety of diseases including cancer. PDT based on upconversion nanoparticles (UCNPs) has received much attention in recent years. Under near-infrared (NIR) light excitation, UCNPs are able to emit high-energy visible light, which can activate surrounding photosensitizer (PS) molecules to produce singlet oxygen and kill cancer cells. Owing to the high tissue penetration ability of NIR light, NIR-excited UCNPs can be used to activate PS molecules in much deeper tissues compared to traditional PDT induced by visible or ultraviolet (UV) light. In addition to the application of UCNPs as an energy donor in PDT, via similar mechanisms, they could also be used for the NIR light-triggered drug release or activation of 'caged' imaging or therapeutic molecules. In this review, we will summarize the latest progresses regarding the applications of UCNPs for photodynamic therapy, NIR triggered drug and gene delivery, as well as several other UCNP-based cancer therapeutic approaches. The future prospects and challenges in this emerging field will be also discussed.
\end{abstract}

Key words: Photodynamic therapy, Upconversion nanoparticles.

\section{Introduction}

In the past few decades, photodynamic therapy (PDT) has emerged as alternative treatment approach to chemotherapy and radiotherapy to treat various diseases including cancer. PDT is considered to be minimally invasive compared with chemotherapy and radiotherapy.[1] Three key components are involved in a typical PDT process: light, photosensitizer (PS) molecules, and oxygen. With the absorption of light, a ground state PS is boosted into a high-energy state, leading to energy transfer to neighboring oxygen or other substrate molecules, and then the generation of singlet oxygen $\left({ }^{1} \mathrm{O}_{2}\right)$ or other reactive oxygen species (ROS). The ROS species produced during PDT are able to destroy tumors by multifactorial mechanisms, including directly inducing cancer cell death by necrosis and/or apoptosis,[2] destruction of tumor vasculatures as an anti-angiogenesis effect,[3] and also the stimulation of the host immune system to recognize, track down and destroy any remaining tumor cells, in contrast to radiotherapy and chemotherapy which are mostly immunosuppressive.[4] Moreover, in common with other local cancer therapies such as cryotherapy and hyperthermia, PDT can be locally applied onto a specific region by selectively illuminating the lesion such as the tumor by light, while leaving normal tissues untouched, thus offering much lower toxic side effects compared to the radiotherapy and chemotherapy which kill normal cells together with cancer cells in a less selective manner.

Despite the above-mentioned advantages of PDT, one major limitation of this treatment methodology in clinical applications is the poor tissue pene- 
tration ability of the light used to activate PS molecules. In fact, most PS molecules currently used in PDT are excited by visible or even UV light, which exhibit rather limited penetration depth in biological tissues, thus hampering the application of PDT in the treatment of large or internal tumors. Therefore, significant efforts have been devoted to develop new PDT strategies induced by NIR light, whose wavelengths fall into the 'transparency window' of biological tissues. It is believed that NIR light (typically in the 700-1000 nm spectral range) not only can afford penetration depths of an order of magnitude greater than that of visible light, but also with a low phototoxicity to normal cells and tissues, and thus is the ideal light source in phototherapies.[5, 6]

Two-photon excited PDT is a new technique developed in recent years that uses NIR light to induce PDT.[7-10] In this approach, a PS molecule, which usually has to be excited by high-energy visible light, can be excited by simultaneous absorption of two NIR low energy photons into the high-energy state to trigger single oxygen generation. However, this technique requires the use of a pulsed laser as the light source to excite focused small areas to obtain sufficient instant energy that needed for two-photon excitation, and thus has limited value for in vivo experiments, not to mention clinical applications.

Another new approach to deliver light into deeper tissues for PDT treatment is using NIR-excitable upconversion nanoparticles (UCNPs) as an energy donor.[11, 12] UCNPs are usually lanthanide-doped nanocrystals, which emit high energy photons under excitation by the NIR light, and have found potential applications in many different fields including biomedicine. A number of different approaches, mainly including thermal decomposition method and hydrothermal method, have been developed to synthesize UCNP nanocrystals with varied sizes and emission spectra[13]. Compared with traditional down-conversion fluorescence, the NIR light excited upconversion luminescence (UCL) of UCNPs exhibits improved tissue penetration depth, higher photochemical stability, and free of auto-fluorescence background[13-22]. Owing to those unique advantages, UCNPs have been widely explored as novel nano-probes in biomedical imaging in recent years. The absence of auto-fluorescence background in UCL of UCNPs offers remarkably improved detection sensitivity, beneficial for highly sensitive in vivo biomedical imaging and cell tracking[23-27]. The UCL emission spectra could be easily tuned via either changing of doping lanthanide ions, or luminescence resonance energy transfer (LRET) by attaching fluorescent dyes or quantum dots (QDs)[28-30]. By de- veloping UCNPs with multiple imaging functions, the UCL imaging may be combined with other imaging modalities such as magnetic resonance (MR) imaging, positron emission tomography (PET), and computer tomography (CT), for multimodal biomedical imaging using a single UCNP-based imaging probe, to overcome the limitations of each single imaging methodology.[31-36] Moreover, therapeutic applications of UCNPs, especially UCNP-based PDT, have also aroused significant interests recently.

Different from the two-photon excited PDT which requires the pulsed laser with high instant energy as the excitation light and can only treat small areas using the focused laser beam, continuous lasers with much lower energy density can be applied to illuminate large areas in UCNP-based PDT. In the past few years, a large number of groups have demonstrated UCNP-based PDT in vitro as well as in vivo. In addition, UCNPs as energy donors can be used for the NIR light-triggered release or 'uncaging' of functional bio-molecules. Several other UCNP-based therapeutic approaches, including imaging-guided drug delivery and photothermal therapy, have also been presented by several different groups. Although there have been a number of recently published articles to review the use of UCNPs in biomedicine, most of them are focused on their synthesis, functionalization, as well as applications in biological sensing and imaging[13, 16, 19]. In this article, while mainly reviewing the development of UCNP-based PDT in details, we will also summarize recent reports regarding the involvements of UCNPs in other cancer therapeutic applications, and discuss the future challenges and promises of using UCNPs as novel theranostic nano-platforms.

\section{Loading of photosensitizers on upcon- version nanoparticles}

Appropriate loading of PS molecules on to UCNPs is important to allow effective resonance energy transfer from donors to acceptors in NIR-induced PDT using UCNP-PS nanocomplexes. Surveying literature, there have been three commonly used approaches to load PS molecules onto UCNPs, including silica encapsulation, non-covalently physical adsorption, and covalent conjugation via chemical linkages (Table 1).

\section{I Silica encapsulation}

The first report of using UCNPs for PDT application was published by Zhang et al.[37] In their study, UCNPs were coated with a silica shell, into which PS molecules were doped. Since then, a number of papers have reported similar strategies for the 
PS loading on UCNPs.[38-46] The team of Zhang [46] developed $\mathrm{NaYF}_{4}$ UCNPs as carriers for zinc(II)-phtalocyanine ( $\mathrm{ZnPc}$ ) (Figure 1a). Instead of doping PS molecules into the silica shell during nanoparticle synthesis, in their system, UCNPs were coated with a layer of mesoporous silica, into which $\mathrm{ZnPc}$ molecules were encapsulated by physical adsorption. They demonstrated that the $\mathrm{ZnPc}$ molecules loaded into the porous silica shell of nanoparticles were quite stable without being released in phosphate-buffered saline (PBS) solution and RPMI 1640 cell culture medium. Under 980-nm NIR laser excitation, those nanoparticles could continuously produce singlet oxygen for PDT cancer cell killing. This silica encapsulation strategy, although demands certain efforts in nanomaterials fabrication, is likely the most widely adopted method to prepare UCNP-based PDT agents.

\subsection{Non-covalent physical adsorption}

Non-covalent physical adsorption, as a very simple method to load PS molecules on UCNPs, has been proposed by our group.[47, 48] Since as-prepared UCNPs were capped by oleic acid and not water soluble, a polyethylene glycol (PEG) drafted amphiphilic polymer was used to transfer hydrophobic UCNPs into the aqueous phase, yielding PEGylated UCNPs with excellent water solubility. PEGylated UCNPs prepared by our method contained a hydrophobic oleic acid layer on top of the inorganic nanoparticle surface and beneath the PEG coating. Hydrophobic molecules such as de-protonated doxorubicin (DOX), a chemotherapy drug, and chlorine 6 (Ce6), a PS molecule, could thus be adsorbed into the hydrophobic layer for effective drug loading. The terminals on PEG chains were available for conjugation of targeting ligands such as folate acid for targeted delivery. Our strategy is rather straightforward without the need of any surface reaction and allows the close binding between the nanoparticle and Ce6 molecules, offering effective resonance energy transfer from UCNPs to Ce6. (Figure 1b)

Table I. A summary of published efforts on the development of UCNP-based PDT.

\begin{tabular}{|c|c|c|c|c|c|}
\hline Author & Size & Coating & Photosensitizer & Congujated PSs to NPs & Ref. \\
\hline Qian et al. & $60-120 \mathrm{~nm}$ & Silica & Merocyanine-540 & Sailica encapsulation & 46 \\
\hline Guo et al. & $60 \mathrm{~nm}$ & silica & Zinc phthalocyanine & Sailica encapsulation & 43 \\
\hline Chatterjee et al. & $50 \mathrm{~nm}$ & PEI & Zinc phthalocyanine & $\begin{array}{l}\text { Non-covalently physical } \\
\text { adsorption }\end{array}$ & 52 \\
\hline Ungun et al. & $280 \mathrm{~nm}$ & PEG & Tetraphenyl porphine & $\begin{array}{l}\text { physical encapsulation or } \\
\text { adsorption }\end{array}$ & 45 \\
\hline Liu et al. & $\sim 20 \mathrm{~nm}$ & $\begin{array}{l}\text { 2-aminoethyl dihydrogen } \\
\text { phos-phate (AEP) }\end{array}$ & Rose bengal & covalent linkage conjugation & 49 \\
\hline Shan et al. & $\sim 100 \mathrm{~nm}$ & PEG & $\begin{array}{l}\text { meso -Tetraphenyl porphine } \\
\text { (TPP) }\end{array}$ & $\begin{array}{l}\text { physical encapsulation or } \\
\text { adsorption }\end{array}$ & 44 \\
\hline Qiao et al. & $22 \mathrm{~nm}$ & silica & $\begin{array}{l}\text { Silicon phthalocyanine } \\
\text { dihydroxide(SPCD) \& } \\
\text { hematoporphyrin(HP) }\end{array}$ & Sailica encapsulation & 41 \\
\hline Zhao et al. & $38 \mathrm{~nm}$ & silica & $\mathrm{AlC}_{4} \mathrm{PC}$ & Sailica encapsulation & 40 \\
\hline Zhou et al. & $53 \mathrm{~nm}$ & PEI-OCMC & Pyropheophorbide & Covalent conjugation & 50 \\
\hline Wang et al. & $30 \mathrm{~nm}$ & PEG & Chlorin e6 & $\begin{array}{l}\text { Non-covalently physical } \\
\text { adsorption }\end{array}$ & 48 \\
\hline Chen et al. & $38 \mathrm{~nm}$ & $\mathrm{SiO} 2$ & methylene blue & Sailica encapsulation & 39 \\
\hline Cui et al. & $55 \mathrm{~nm}$ & amphiphilic SOC & Zinc phthalocyanine & SOC encapsulation & 53 \\
\hline Park et al. & $66.6 \mathrm{~nm}$ & PEG & Chlorin e6 & $\begin{array}{l}\text { Both non-covalently physical } \\
\text { adsorption and covalent } \\
\text { conjugation }\end{array}$ & 51 \\
\hline Idris et al. & $100 \mathrm{~nm}$ & $\mathrm{SiO} 2$ & $\begin{array}{l}\text { merocya-nine } 540 \text { \& Zinc } \\
\text { phthalocyanine }\end{array}$ & Sailica encapsulation & 6 \\
\hline
\end{tabular}




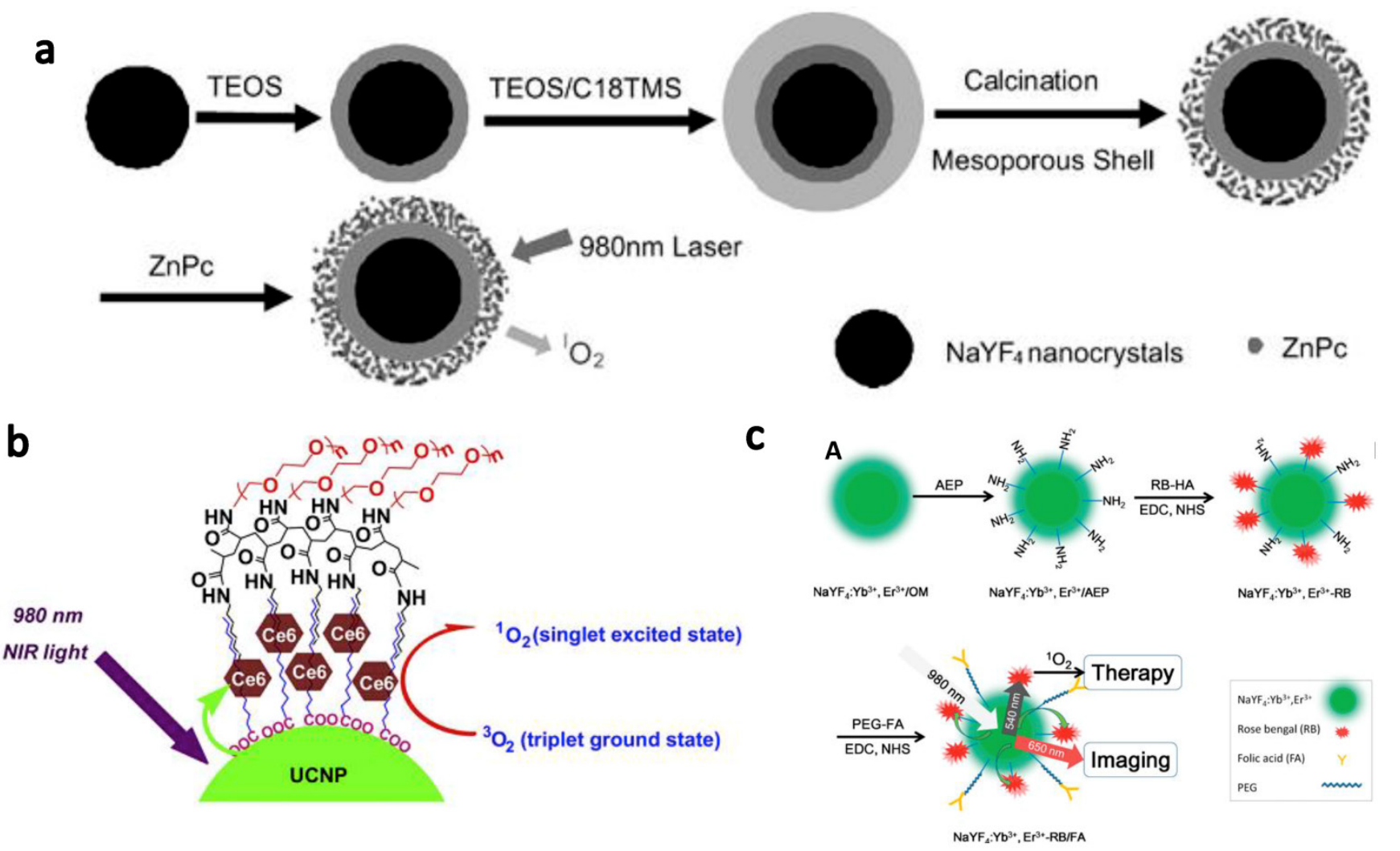

Figure I. Different strategies to load PS molecules on UCNPs. (a) A schematic image showing loading of ZnPc into mesoporous silica shell on $\mathrm{NaYF}_{4} @$ silica nanoparticles by physical encapsulation and use them for PDT. (b) A schematic drawing showing Ce6 physically adsorbed on the surface of PEGylated UCNPs via hydrophobic interactions to form UCNP-Ce6 complex. c) Covalent conjugation of photosensitizer RB and target molecule FA onto amine functionalized UCNPs. Copyright 2009 Wiley,[46] 2012 Elsevier[48] and 2012 American Chemical Society.[49]

a

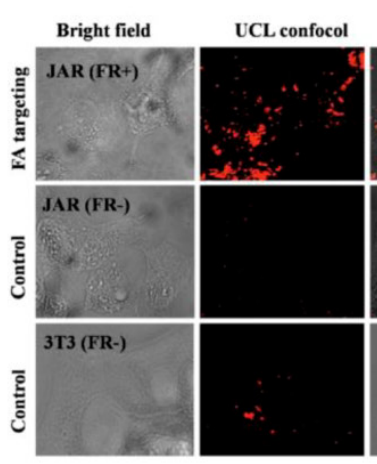

Merged

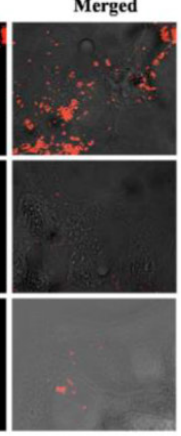

b

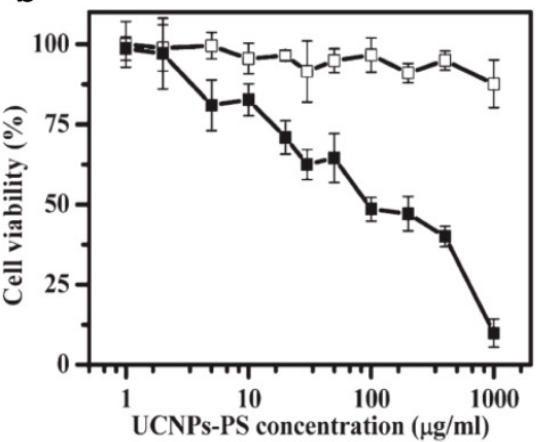

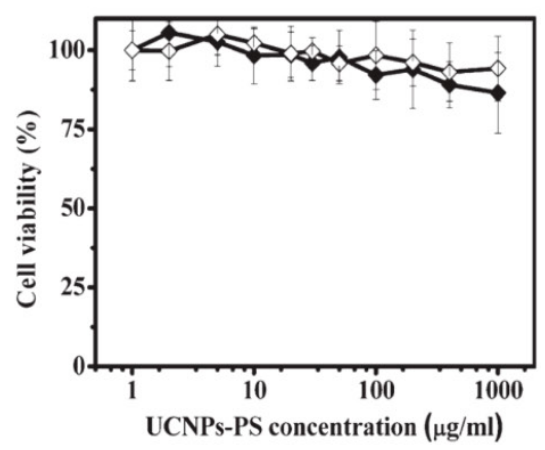

Figure 2. In vitro targeted PDT using UCNPs. (a) Confocal UCL images showing specific targeting of FR positive JAR carcinoma cells by UCNP-RB-FA. (b) Relative viabilities of JAR cells (left, FR+) and NIH 3 T3 fibroblasts (right, FR-) treated with UCNP-RB-FA at different concentrations with (solid) or without (open) 980-nm laser exposure. Excellet FR+ cancer cell targeting effect was evidenced by both confocal UCL images and selective PDT killing effects. Copyright 2012 American Chemical Society.[49]

\subsection{Covalent conjugation.}

When physically entrapped inside the silica network or the hydrophobic layer, those PS molecules may be prematurely released from the carrier which can lead to a reduced efficiency of treatment. Covalent coupling of PS molecules inside or at the surface of nanoparticles may be able to overcome these drawbacks. Zhang et al.[49] described a covalent bonding strategy to link a PS molecule, Rose Bengal (RB), onto UCNPs (Figure 2), by conjugating carboxyl groups on hyaluronic acid (HA) modified RB to amino groups on the surface of UCNPs via amide bonds. They found the covalent bonding between UCNPs and RB molecules significantly improved the PS loading capacity and the energy transfer efficiency from nanoparticles to PS molecules. In another work, Zhou et al.[50] used O-carboxymethylated chitosan to modify 
UCNPs. Chemical covalent linkage was employed to conjugate targeting peptide $\mathrm{c}(\mathrm{RGDyK})$ and photosensitizing pyropheophorbide a (Ppa) to UCNPs. They showed that covalently linked Ppa molecules not only were stable but also retained their spectroscopic and functional properties for targeted NIR-induced PDT. (Figure 1c)

In a very recent work, Hyeon and co-workers [51] combined both physical adsorption and chemical conjugation methods to attach PS molecules onto UCNPs. Water-dispersible and amine-functionalized UCNPs encapsulated by PEGylated phospholipids were synthesized. Owing to the hydrophobic property and planar structure of chlorin, Ce6 could be readily entrapped in hydrophobic phospholipid layers surrounding UCNPs. On the other hand, the reaction between the amine-functionalized UCNPs and the carboxy group of $\mathrm{Ce} 6$ was employed to form an amide bond. The amount of Ce6 loading calculated from the UV-vis absorption spectra was found to be > $10{ }^{3} \mathrm{Ce} 6$ molecules per particle.

\section{In vitro PDT using UCNPs.}

In vitro PDT using UCNP-PS nanocomplexes was demonstrated in several early cell experiments.[37, 42, 43, 52] In a study by Zhang and co-workers,[43] mesoporous silica coated $\mathrm{NaYF}_{4}(\mathrm{Yb}: \mathrm{Er})$ UCNPs core-shell nanoparticles were loaded with ZnPc. Upon irradiation at $980 \mathrm{~nm}$, visible light emitted from UCNPs could activate ZnPc molecules in the silica shell via resonance energy transfer, showing a strong photodynamic cell destruction effect on MB49 cells. DNA ladder fragments were found after PDT treatment of MB49 cells, together with cytochrome $c$ release from the mitochondria into the cytosol. These data showed that DNA bases and mitochondria were attacked by reactive singlet oxygen molecules, which were generated during NIR laser irradiation. A laser power at $0.5 \mathrm{~W}$ and an irradiation time of $5 \mathrm{~min}$ were needed to induce significant cell death in this work. In another report, Shan et al.[44] increased the loading ratio of photosensitizers molecules on UCNPs to be about $10 \mathrm{wt}$. \% by encapsulating $100 \mathrm{~nm}$ UCNPs and meso-tetraphenyl porphine photosensitizers in poly(ethylene glycol-block-(DL)lactic acid). The composite nanoparticles were shown to exhibit efficient HeLa cervical cancer cell-killing activity upon 980-nm NIR excitation at $134 \mathrm{~W} / \mathrm{cm}^{2}$ for $45 \mathrm{~min}$, which, however, was an extremely high optical dosage.

UCNP for targeted NIR photodynamic therapy of cancer cells has also been reported by several groups. Liu et al.[49] used folic acid (FA) to functionalized UCNP-RB nanoconjugates. A covalent bonding strategy was used to construct a highly efficient NIR photosensitizing nanoplatform for simultaneous PDT and imaging. Specific targeting of folate receptor (FR) positive JAR carcinoma cells was evidenced by UCL cell imaging. After NIR laser irradiation at $1.5 \mathrm{~W} / \mathrm{cm}^{2}$ for $10 \mathrm{~min}$, cell viabilities decreased significantly for the JAR carcinoma cells but were not obviously changed for FR negative noncancerous NIH 3T3 cells (Figure 2). Zhou et al.[50] used a photosensitizer pyropheophorbide a (Ppa) and RGD peptide c(RGDyK) co-modified chitosan-wrapped NaYF4:Yb/Er UCNPs for targeted PDT. Selective PDT killing of integrin $\mathrm{a}_{\mathrm{v}} \beta_{3}$ positive U87-MG tumor cells was demonstrated by using those UCNP-Ppa-RGD conjugates and 980-nm NIR laser irradiation at $300 \mathrm{~J} / \mathrm{cm}^{2}$.

The success of using UCNP-PS nanocomplexes for in vitro PDT cancer cell killing under NIR light thus encourages further investigations of this approach for in vivo cancer treatment, which will be discussed in the following section.

\section{In vivo PDT using UCNPs.}

The first in vivo UCNP-based PDT study in animal experiments was demonstrated by our team (Figure 3).[48] We non-covalently incorporated Ce6 onto PEGylated amphiphilic polymer-coated UCNPs. The obtained UCNP-Ce6 complex could enter cancer cells and induce $4 \mathrm{~T} 1$ cell death after being exposed to the $980-\mathrm{nm}$ light at $0.5 \mathrm{~W} / \mathrm{cm}^{2}$ for $30 \mathrm{~min}$. For animal experiments, we directly injected UCNP-Ce6 (20 $\mathrm{mg} / \mathrm{ml} \mathrm{UCNP}, \sim 1.5 \mathrm{mg} / \mathrm{ml} \mathrm{Ce} 6)$ into $4 \mathrm{~T} 1$ murine breast cancer tumors grown on BABL/C mice. After 980-nm NIR laser excitation for $30 \mathrm{~min}$ (with 1-min interval for each minute to avoid heating) at $0.5 \mathrm{w} / \mathrm{cm}$ 2 , excellent tumor regression effect was observed for UCNP-Ce6 injected tumors, while neither UCNP-Ce6 injection by itself nor NIR-laser irradiation alone was able to delay the tumor growth (Figure 3a). The survival time of mice after UCNP-Ce6 injection and PDT treatment was dramatically pro-longed compared to the control mice as well (Figure 3b). Moreover, we found that UCNPs injected into tumors after PDT treatment could be gradually cleared out from mouse organs after 2 months as determined by ex vivo inductively coupled plasma-atomic emission spectrometry (ICP-AES) assay (Figure 3c), without obviously rendering noticeable toxicity to the treated animals. At last, we also compared the tissue penetration abilities between UCNP-based PDT induced by $980-\mathrm{nm}$ NIR light and traditional PDT using visible light at $660 \mathrm{~nm}$, by blocking irradiated subjects using pork tissues with different thicknesses. Although direct exposure of a Ce6 solution to the 660-nm light generated much more singlet oxygen compared to 
UCNP-Ce6 sample under the 980-nm excitation (the same Ce6 concentration, the same optical power density), ${ }^{1} \mathrm{O}_{2}$ formation of the free $\mathrm{Ce} 6$ sample was dramatically reduced if the $660-\mathrm{nm}$ light was blocked by a 3-mm thick pork tissue and completely eliminated if blocked by a 8-mm thick tissue. In contrast, the ${ }^{1} \mathrm{O}_{2}$ production remained a decent level for UCNP-Ce6 even when the $980-\mathrm{nm}$ light was blocked by a $8-\mathrm{mm}$ thick tissue. In vitro and in vivo experiments further confirmed that compared to the traditional PDT relying on visible light excitation, the NIR light triggered PDT of cancer using UCNP-Ce6 nanocomplex offers terrifically improved tissue penetration depth and could be preferable for the treatment of large or internal tumors.
In another work, Cui et al.[53] developed amphiphilic chitosan modified UCNPs loaded with ZnPc for UCNP-based PDT in vivo. Hydrophobic OA-capped UCNPs were modified with biodegradable $\mathrm{N}$-succinyl-N'-octyl chitosan (SOC) to improve biocompatibility of UCNPs. ZnPc was loaded into SOC-UCNPs with high drug loading capacity through hydrophobic interactions with the SOC layer. Via intra-tumoral injection, a prominent anti-tumor effect of the ZnPc-UCNPs to S180 tumors on mice was observed after 980-nm NIR laser irradiation at 0.4 $\mathrm{W} / \mathrm{cm}^{2}$ for $15 \mathrm{~min}$. Their results are consistent with our work [48] and offer another example of in vivo UCNP-based PDT treatment in animal studies.
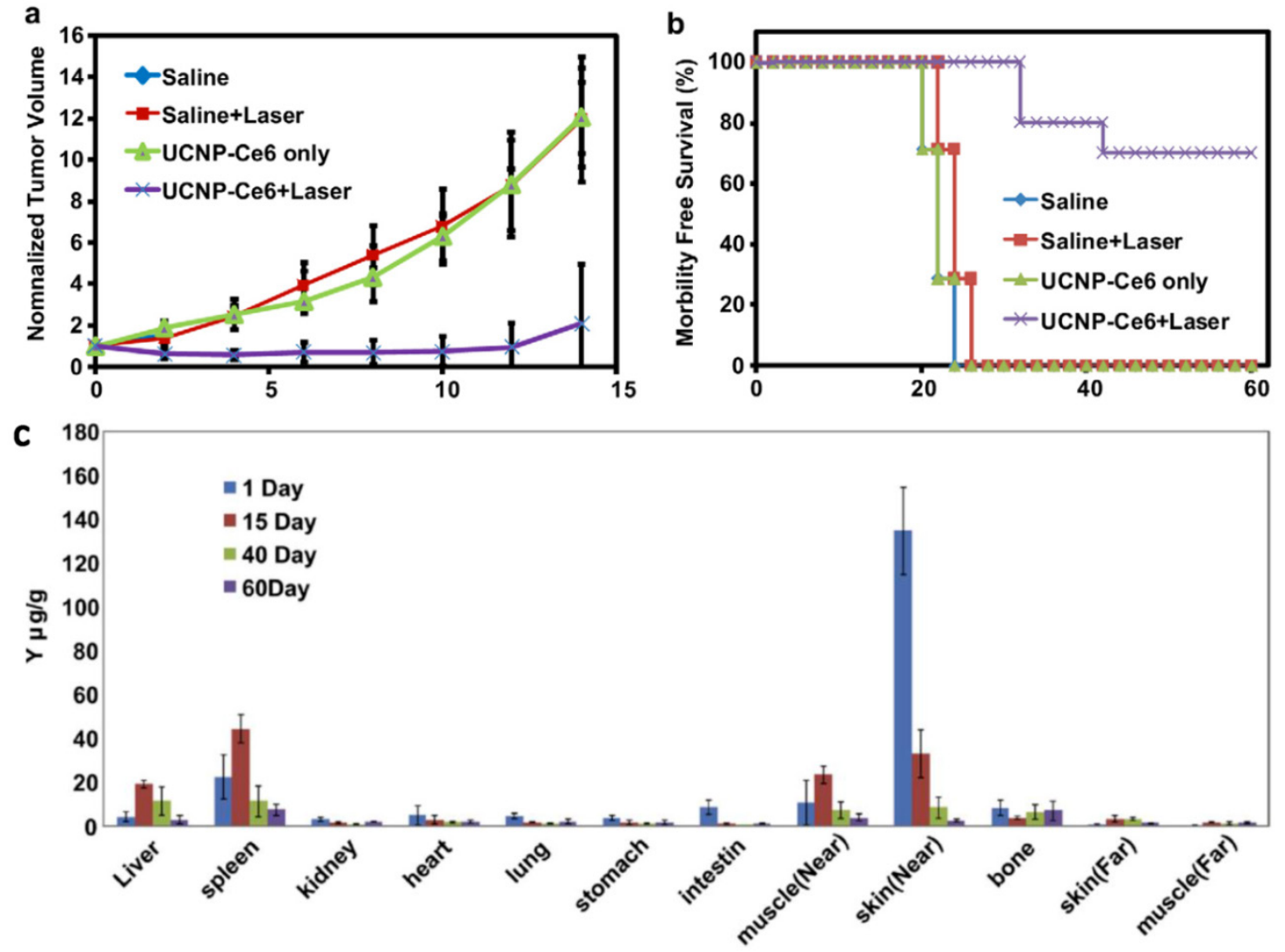

Figure 3. In vivo PDT treatment of tumor-bearing mice by UCNP-Ce6. (a) The growth of 4TI tumors on different groups of mice after various treatments indicated. (b) The survival curves of mice in 60 days after various treatments indicated. (c) Biodistribution of Yttrium in various organs of UCNP-Ce6 injected mice after PDT treatment. ICP-AES was employed to quantitatively determine the $\mathrm{Y}^{3+}$ levels. Our work demonstrated NIR light-induced in vivo PDT treatment of s.c. tumor in animals, and UCNPs would be cleared out from the mouse body after 2 months. Copyright 2012 Elsevier.[48] 

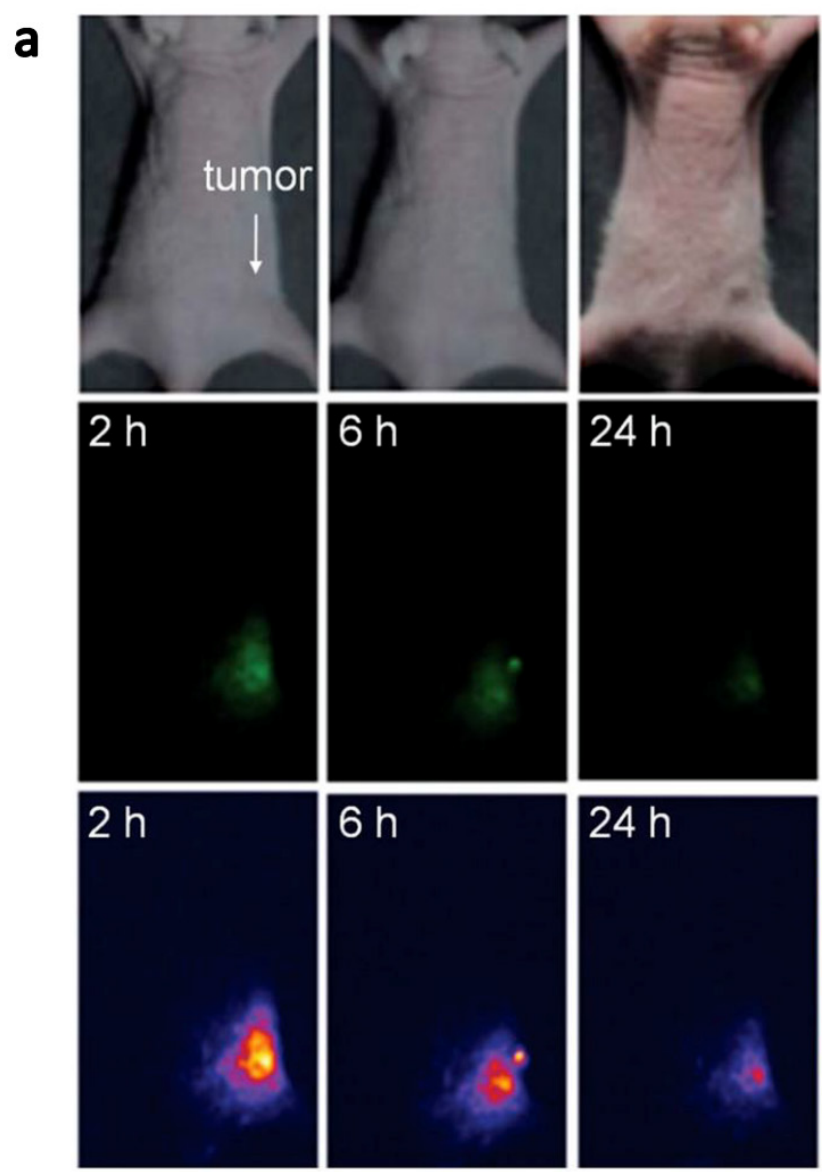
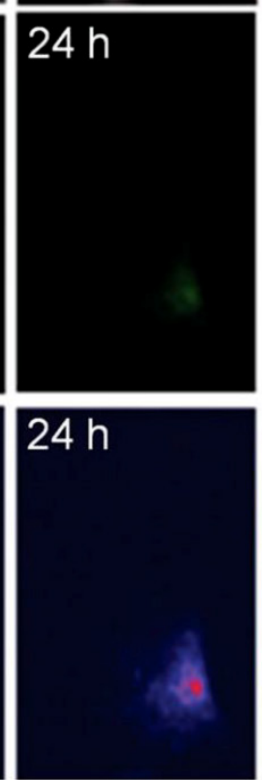

b
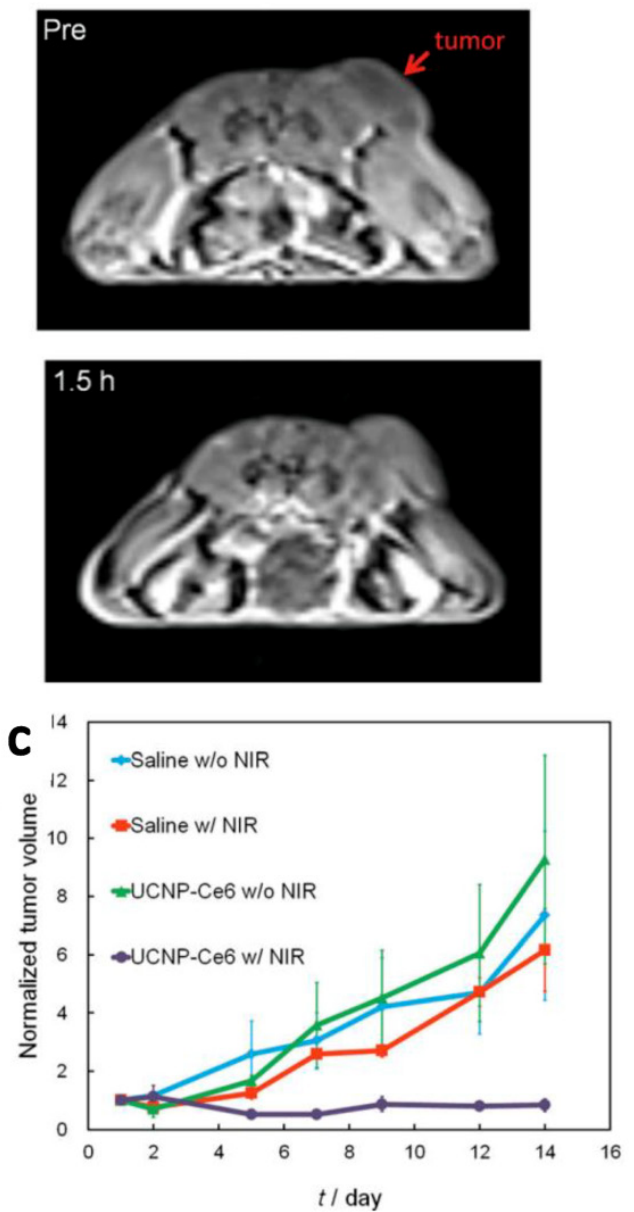

Figure 4. In vivo imaging-guided PDT. (a) UCL images of nude mice bearing tumors after intravenous injection of UCNP-Ce6. (b) TI-weighted MR images of a tumor-bearing nude mouse before and after $1.5 \mathrm{~h}$ intravenous injection of UCNP-Ce6. (c) Growth of tumors after various treatments indicated for efficient imaging guided PDT therapy. Copyright 2012 Wiley-VCH.[5I]

Very recently, Hyeon and co-workers [51] reported in vivo PDT effect through the systemic administration of UCNP-Ce6 followed by the 980-nm irradiation (Figure 4). $\mathrm{NaGdY}_{4}$-based UCNPs after PEGylation were loaded with Ce6 molecules by both physical adsorption and chemical conjugation, yielding a UCNP-Ce6 complex with $>10^{3}$ Ce6 molecules per particle. The blood circulation half-life of UCNP-Ce6 was determined to be $21.6 \mathrm{~min}$ in BABL/C mice after intravenous injection. Nude mice bearing U87MG tumors were injected with UCNP-Ce6 through the tail vein $(0.1 \mathrm{mg}$ of rare earth per mouse). Obvious tumor accumulation of UCNP-Ce6 nanoparticles was revealed by dual-modal upconversion luminescence imaging and T1-weighted MR imaging (Figure 4a\&b), and could likely be attributed to the enhanced permeability and retention (EPR) effect of cancerous tumors. Under the $980 \mathrm{~nm}$ irradiation, tumor growth of UCNP-Ce6 injected mice was significantly inhibited compared with other control groups (Figure 4c). These results clearly indicate the great potential of using UCNPs for multi-modal imaging guided PDT. Remarkably, this study is the first report to demonstrate UCNP-based in vivo PDT through the systemic administration of UCNP-PS complexes.

In another latest report, Zhang and coauthors demonstrated in vivo tumor-targeted PDT using UCNPs.[6] $\mathrm{NaYF}_{4}(\mathrm{Yb}$ :Er) UCNPs were coated with mesoporous silica and co-loaded with $\mathrm{ZnPc}$ and merocya-nine 540 (MC540), which were two widely used PS molecules with different excitation wavelengths (Figure 5). Two main peaks, green $(\sim 540 \mathrm{~nm})$ and red $(\sim 660 \mathrm{~nm})$ emissions of UCNPs excited by 980-nm laser matched well with the absorption of two types of PS molecules, for fully utilization of upconverted energy to maximize the PDT efficiency. ZnPc/MC540 coloaded UCNPs resulted in the high rate to generate ${ }^{1} \mathrm{O}_{2}$ in the solution as well as in the live cells under the NIR light treatment. Then, they carefully assessed the therapeutic efficacy of those $\mathrm{ZnPc} / \mathrm{MC5} 40$ coloaded UCNP-PS complexes as an in 
vivo PDT agent in three sets of mouse experiments. In the first step, they injected B16-F0 melanoma cells labeled with $\mathrm{ZnPc} / \mathrm{MC} 540$ coloaded UCNPs under the skin of C57BL/ 6 mice and then irradiated the injected site with a $980-\mathrm{nm}$ laser $\left(415 \mathrm{~mW} / \mathrm{cm}^{2}, 2 \mathrm{~h}\right)$, observing sounded PDT efficacy. After that, they further confirmed the PDT efficacy by intratumorally injecting $\mathrm{ZnPc} / \mathrm{MC5} 40$ coloaded UCNPs into C57BL/ 6 mice bearing melanoma tumors. Great tumor regression effect was also noticed after the injected tumors were exposed to the NIR laser. At last, they further examined the targeted PDT efficacy using PEGylated UCNPs conjugated with folic acid. Mice bearing B16-F0 melanoma tumors were intravenously injected with ZnPc/MC540 coloaded FA-PEG-UCNPs

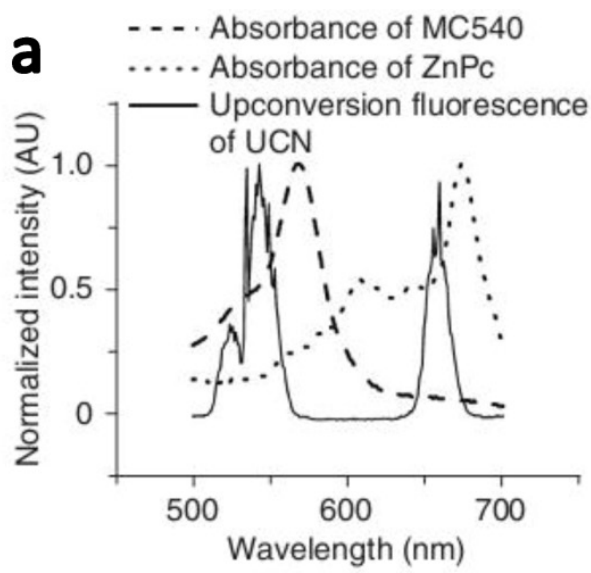

b
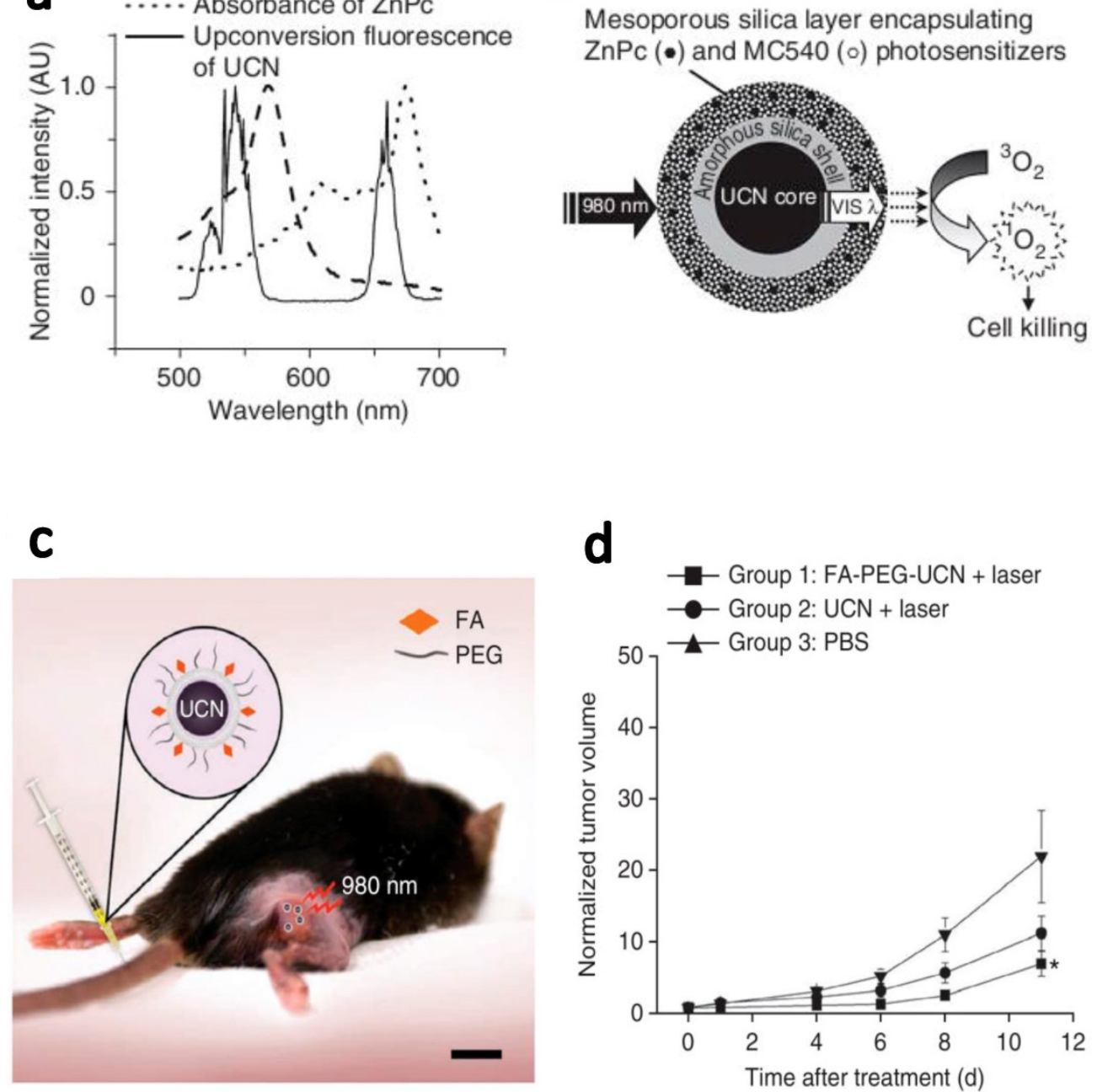

Figure 5. Targeted in vivo PDT of a subcutaneous tumor model injected with FA-PEG-UCNPs. (a) The fluorescence emission spectrum of UCNPs under 980-nm NIR laser excitation and the absorption spectra of ZnPc and MC540 photosensitizers. (b) A schematic drawing showing mesoporous-silica-coated UCNPs coloaded with ZnPc and MC540 for PDT. (c) A schematic diagram showing UCNP-based targeted PDT in a mouse model of melanoma. Those ZnPc/MC540 co-loaded UCNPs were functionalized with PEG and conjugated with FA for in vivo tumor targeting. (d) Change in tumor size as a function of time after various treatments indicated. Intravenously injected FA-PEG-UCNPs co-loaded with ZnPc/MC540 was able to obviously delay the tumor growth after the 980-nm NIR laser exposure. Copyright 2012 Nature Publishing Group.[6] and then irradiated with a 980-nm laser at $4 \mathrm{~h}$ post injection. A significant reduction in tumor growth of the treatment group was observed compared to control mice treated with PBS (Figure 5). This is the first time that actively tumor-targeted PDT using UCNPs is demonstrated in vivo.

As discussed above, UCNP-based PDT has been successfully demonstrated in vivo, showing encouraging therapeutic effect upon either local injection or systemic administration of UCNP-PS nanocomplexes. Further efforts in this field may enable a new photodynamic therapeutic approach with greatly improved tissue penetration, suitable for treatment of relatively large or internal tumors. 


\section{NIR triggered drug release and gene delivery using UCNPs.}

In addition to the application of UCNPs in PDT, they could also be used as an energy donor to trigger photochemistry that usually happens under UV or visible light by NIR light irradiation.[5, 54-57] Photo responsive drug release systems have received significant interests in recent years, owing to their great potential in remotely controlled release of therapeutics at the site of interest (e.g. tumors).[58] However, the majority of photochemistry that employed in photo responsive drug release systems requires high-energy UV excitation, which not only induces substantial phototoxicity, but also exhibits short penetration depth in biological systems. A specific class of UCNPs, usually containing $\mathrm{Yb}$ and $\mathrm{Tm}$, are able to emit UV light under 980-nm NIR excitation, and could have great promise in the design of NIR-light responsive drug delivery systems.

In 2010, Carling et al.[55] for the first time demonstrated the use of NIR exciting \& UV emitting (NIR-to-UV) UCNPs ( $\mathrm{NaYF}_{4}: \mathrm{Yb} \mathrm{Tm}$ ) for NIR laser triggered molecular releasing. A caged compound, 3', 5'-di(carboxymethoxy)benzoin, which was usually photo activated by UV light, was conjugated to NIR-to-UV UCNPs. Under NIR laser irradiation, UV light generated by UCNPs could trigger the un-caging process and result in the release of organic molecules, a carboxylic acid in this case, from UCNPs. In a later work, Yan et al. synthesized a UV-light responsive amphiliphic block co-polymer to co-encapsulate hydrophobic NIR-to-UV UCNPs by micelle formation.[56] When exposed to the NIR laser, UCNPs could emit UV light, which then could cleave the photo-labile hydrophobic o-nitrobenzyl groups in this co-polymer, thus resulting in the dissociation of micelles and release of co-loaded hydrophobic molecular payloads. Although these two systems are just proof-of-concept studies, the strategy presented in their reports is expected to be adopted for the design of NIR light controllable drug release platforms based on UCNPs.

Recently, Zhang and co-workers[54] reported the use of NIR-to-UV UCNPs for photo-controllable gene expression (Figure 6). Plasmid DNA encoding green fluorescence protein (GFP) and small interfering RNA (siRNA) target GFP mRNA were both caged with 4,5-dimethoxy-2-nitroacetophenone DMNPE to block their respective functions. After NIR light treatment, they were uncaged by the energy transferred from
UCNPs, inducing controlled gene expression and specific gene silencing, respectively. The NIR-to-UV UCNPs overcome the drawback of current photo-responsive systems in which UV light is needed to activate DMNPE caged nucleic acids. Besides, the upconverted UV produced by the irradiated UCNPs was also found to be relatively safe for the cells, under the applied nanoparticle dosage and duration of NIR laser irradiation. This system was then further used to activate photocaged nucleic acids in animal models. Cells transfected with UCNPs containing photocaged GFP plasmid were loaded into a polydimethylsiloxane (PDMS) device, which was transplanted into mice. Efficient GFP activation was observed even at the deep tissues. Those results prove that this technique has enormous potential in a number of fields including gene therapy for controlled and specific gene delivery/knockdown, developmental biology for site-specific gene knockdown, and patterning of biomolecules using safe NIR light.

Bioluminescence imaging is a technology that allows for the noninvasive study of ongoing biological processes in small laboratory animals. Xing and co-workers [5] reported NIR light controlled uncaging of d-luciferin and bioluminescence imaging in vivo using NIR-to-UV UCNP probes. The core-shell NIR-to-UV UCNPs were coated with thiolated silane molecules and subsequently coupled to d-luciferin that was caged with a 1-(2-nitrophenyl)ethyl group. UV light emitted from UCNPs under NIR irradiation could activate caged d-luciferin to release d-luciferin molecules which was an active substrate of luciferase used in bioluminescence imaging. Cell viability assays showed no obvious cytotoxicity for C6 glioma cells treated with the d-luciferin/UCNP conjugate after two hours of irradiation with NIR light. In marked contrast, UV irradiation resulted in significant cellular damage after a short exposure time. Importantly, strong bioluminescence signals were detected in the mouse injected d-luciferin/UCNP conjugate after NIR-light induced photo uncaging. While under UV irradiation, no notable bioluminescence was detected in the mouse owing to the poor tissue penetration of UV light (Figure 7).

It is foreseeable that UCNPs might find future application as nanotransducers for highly controllable NIR photo-triggered activation of caged cancer theranostics, providing an effective route to develop spatially and temporally controlled drug delivery and imaging systems. 

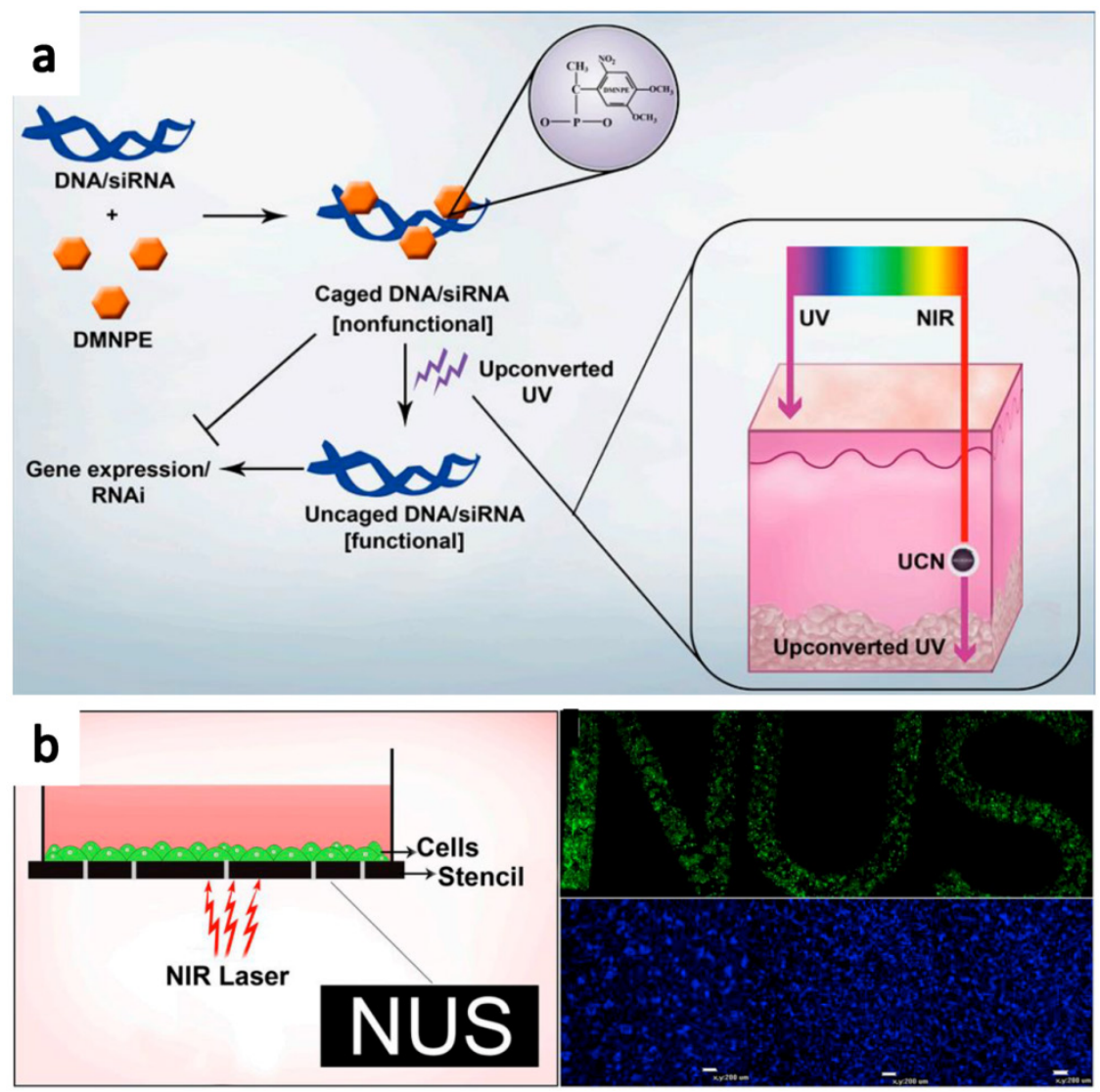

Figure 6. Schematic illustration of NIR triggered gene release using NIR-UV UCNPs. (a) Plasmid DNA or siRNA are caged with DMNPE and then uncaged by upconverted UV light emitted from NIR-to-UV UCNPs. Inset shows the penetration depth of UV and NIR light in the skin. (b) Photoactivation and patterned activation of caged GFP nucleic acids in cells. Copyright 2012 Highwire press PNAS.[54]
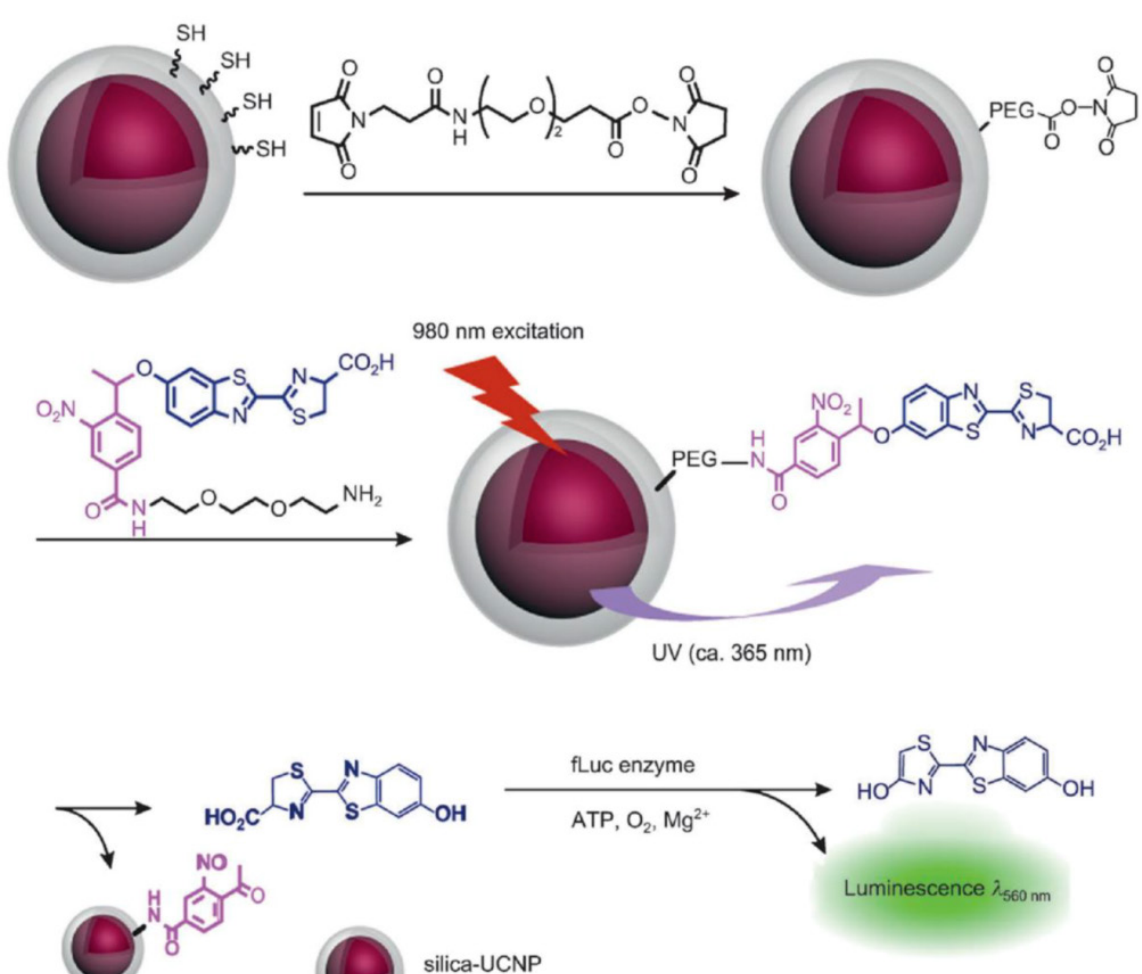

Figure 7. Experimental design for NIR activated bioluminescence imaging. Caged luciferin conjugated on NIR-to-UV UCNPs was activated after 980-nm laser irradiation, releasing un-caged luciferin as an active substrate in bioluminescence imaging. Copyright $2012 \mathrm{Wiley-}-\mathrm{VCH}$.[5] 


\section{Other therapeutic applications and imaging-guided cancer therapies using upconversion nanoparticles and their nanocomposites}

Recently, UCNPs combined with other functionalities have also been explored for multifunctional biomedical imaging and drug delivery.[47, 59-69] In our study, we showed that UCNPs could be loaded with chemotherapy drug molecules for imaging and therapy simultaneously.[47] Doxorubicin (DOX) was loaded on PEGylated UCNPs by physical adsorption and could be released under slightly acidic $\mathrm{pH}$. Utilizing a folate targeting model, targeted cancer cell imaging and drug delivery was achieved. In another recent work, Lin and coauthors[64] reported a drug delivery system based on DOX-conjugated NaYF4: $\mathrm{Yb}^{3+} / \mathrm{Tm}^{3+}$ UCNPs, which was also able to release DOX in mildly acidic environments. The LRET between UCNPs and DOX could be utilized as the optical indicator to monitor the release of DOX inside cells.

In our another work, we encapsulate UCNPs together with iron oxide nanoparticles(IONPs), fluorescent dye and/or anti-cancer drug molecules by using an amphiphilic block copolymer, poly(styreneblock-allyl alcohol) ( $\left.\mathrm{PS}_{16}-\mathrm{b}-\mathrm{PAA}_{10}\right)$, via a microemulsion method, to obtain a multi-functional nanocomposite system(Figure 8a).[65] Triple-modal upconversion luminescence (UCL )/down-conversion fluorescence (FL)/magnetic resonance (MR) imaging was demonstrated in vitro and in vivo, and also applied for in vivo cancer cell tracking in mice. In addition, after loading anti-cancer drug, DOX, into the complex, a novel imaging-guided and magnetic targeted drug delivery system was fabricated for potential multimodal biomedical imaging and cancer therapy.
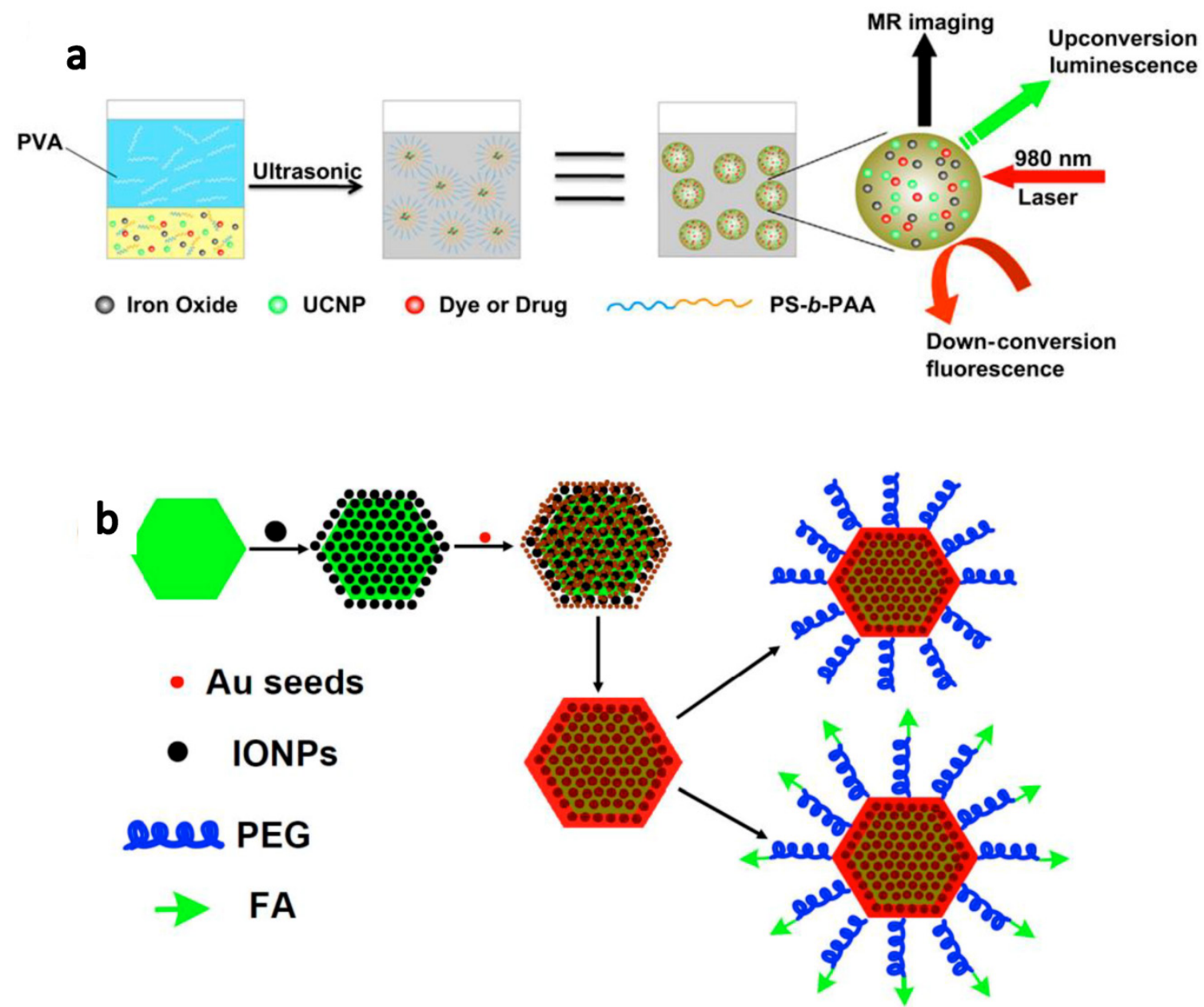

Figure 8. Development of UCNP-based nanocomposites for multifunctional biomedical imaging and therapy. (a) A schematic illustration for UCNP-IONP@Polymer nanocomposite synthesis and applications. (b) A schematic illustration showing UCNP@IONP@Au MFNP synthesis and functionalization. Copyright 20II Elsevier[65] and 20II Wiley-VCH.[62] 
Different from PDT, photothermal therapy is another type of photo therapies that utilizes NIR absorbing agents to generate heat from absorbed light to 'cook' cancer by high temperature. In our group, we developed a novel class of multifunctional nanoparticles (MFNPs) based on UCNPs with combined optical and magnetic properties useful in multimodality imaging and therapy. $[62,63]$ The MFNPs were prepared by a layer-by-layer (LBL) self-assembly strategy. Firstly, ultra-small IONPs were adsorbed on the surface of a UCNP by electrostatic attraction. A thin gold shell was formed by seed-induced reduction growth on the UCNP-IONP complex, yielding UCNP@IONP@Au MFNPs which were then coated by lipoic acid modified PEG to impart stability in physiological solutions. The obtained PEGylated MFNPs were successfully used for in vivo dual-modal imaging guided \& magnetically targeted PTT. Owing to the strong NIR optical absorption of those $\mathrm{Au}$ shelled MFNPs and the highly efficient magnetic tumor targeting, tumors on mice after MFNP-injection and magnetic tumor-targeting were completely eliminated upon exposure to the 808-nm NIR laser (Figure $8 b)$.

Thus, the rapid development in controllable nanomaterials synthesis as well as finely tuned surface engineering could allow researchers to design and fabricate various UCNP-based nanocomposites with highly integrated functionalities, useful for multimodal imaging and imaging-guided cancer therapies.

\section{Conclusions and perspective}

The past decade has witnessed an explosion of interest in the use of UCNPs as novel theranostic probes in biomedicine. As summarized in this review article, applications of UCNPs for photodynamic therapy, NIR triggered drug and gene delivery, as well as several other UCNP-based cancer therapeutic approaches have been demonstrated. However, in the near future, there may still be many obstacles to be overcome.

Firstly, brighter UCNPs need to be synthesized so that stronger energy could be transferred to PS for stronger photodynamic effect. The quantum yield of UCL emission of UCNPs is mostly less than $1 \%$, thus limiting the use of these nano-probes in optical imaging and photodynamic therapy.[70] Tremendous efforts are still needed to frabricate new types of UCNPs with high quantum yield, by designing special UCNP structures (e.g. core-shell structures) [71] and choosing better host matrix materials (e.g. $\left.\mathrm{NaLuY}_{4}\right)$.[72]

Currently, most of UCNPs are excited by the 980-nm light. The imaging penetration depth has been reported to be as thick as $1.6 \mathrm{~cm}$ by $\mathrm{Li}$ and co-workers[26], and $3.2 \mathrm{~cm}$ by Han and co-workers[11]. Although the 980-nm excitation does offer excellent tissue penetration, water also has an absorption peak at around $980 \mathrm{~nm}$, resulting in unnecessary tissue heating during PDT treatment. Development of UCNPs that can be effectively excited by other NIR wavelengths (e.g. $915 \mathrm{~nm}$ as shown by Zhan et al.)[73] may help to solve this problem. Using excitation light at $915 \mathrm{~nm}$ with much lower water absorption only not could avoid skin heating, but may also allow deeper tissue penetration in UCNP-based imaging and PDT treatment.

Better surface functionalization and bioconjugation strategies should also be developed. Most of currently used UCNPs would accumulate in reticuloendothelial systems (RES) such as liver, spleen after systemic administration.[74-76] There is only one example to date to realize active tumor targeted PDT in animals using the folate system,[6] which unfortunately has limited clinical value. There is considerable scope for further development of UCNPs with better surface functionalization to enhance their tumor targeting as well as to reduce their RES retention.

Lastly, before using these UCNPs for clinical therapeutic purposes, overall understanding of nanotoxicology of UCNPs including nanoparticle distribution, excretion, metabolism, pharmacokinetics and pharmacodynamics in animal models should be continually studied in the future. The toxicity of UCNPs is closely associated to their surface chemistry, and biocompatible surface coatings would significantly reduce their cytotoxicity. Current studies have shown that functionalized $\mathrm{NaYF}_{4}$-based UCNPs with biocompatible coatings appear to be not obviously toxic to cells at certain concentration ranges to treated animals over several months.[74-76] However, further in-depth exploration of the physicochemical and physiological processes of UCNPs in vivo is essential such as how this class of nanomaterials would do to animals over an even longer time course, how they interact with immune systems, and whether they may interfere the reproductive system and affect the next generation.

Despite the above challenges, UCNPs with unique optical properties indeed have significant potential in biomedicine. For the imaging, little auto-fluorescence during UCL imaging makes them excellent probes especially for in vivo imaging with extremely high sensitivity (e.g. down to single cell detection limit in animals)[23, 25, 27]. For the NIR-excited PDT or other NIR-triggered theranostics using UCNPs, NIR light offers unmatched advantages 
compared with UV or visible light used in current methodologies. Better design of materials to achieve desired performance and biological behaviors, as well as more in-depth investigation in biomedicine, may further push forward the theranostic applications of UCNPs.

\section{Acknowledgements}

This work was partially supported by the National Basic Research Programs of China (973 Program) (2012CB932600, 2011CB911002), the National Natural Science Foundation of China (51002100), and a Project Funded by the Priority Academic Program Development of Jiangsu Higher Education Institutions.

\section{Competing Interests}

The authors have declared that no competing interest exists.

\section{References}

1. Moan J, Peng Q. An outline of the hundred-year history of PDT. Anticancer Res. 2003; 23: 3591-600.

2. Oleinick NL, Morris RL, Belichenko T. The role of apoptosis in response to photodynamic therapy: what, where, why, and how. Photoch Photobio Sci. 2002; 1: 1-21.

3. Krammer B. Vascular effects of photodynamic therapy. Anticancer Res. 2001; 21: 4271-7.

4. Castano AP, Mroz P, Hamblin MR. Photodynamic therapy and anti-tumour immunity. Nat Rev Cancer. 2006; 6: 535-45.

5. Yang YM, Shao Q, Deng RR, Wang C, Teng X, Cheng $K$, et al. In Vitro and In Vivo Uncaging and Bioluminescence Imaging by Using Photocaged Upconversion Nanoparticles. Angew Chem Int Edit. 2012; 51: 3125-9.

6. Idris NM, Gnanasammandhan MK, Zhang J, Ho PC, Mahendran R, Zhang $Y$. In vivo photodynamic therapy using upconversion nanoparticles as remote-controlled nanotransducers. Nat Med. 2012; doi: $10.1038 / \mathrm{nm} .2933$

7. Gallavardin T, Maurin M, Marotte S, Simon T, Gabudean AM, Bretonniere $\mathrm{Y}$, et al. Photodynamic therapy and two-photon bio-imaging applications of hydrophobic chromophores through amphiphilic polymer delivery. Photoch Photobio Sci. 2011; 10: 1216-25.

8. Velusamy M, Shen JY, Lin JT, Lin YC, Hsieh CC, Lai CH, et al. A New Series of Quadrupolar Type Two-Photon Absorption Chromophores Bearing 11,12-Dibutoxydibenzo[a,c]-phenazine Bridged Amines; Their Applications in Two-Photon Fluorescence Imaging and Two-Photon Photodynamic Therapy. Adv Funct Mater. 2009; 19: 2388-97.

9. Qian J, Wang D, Cai FH, Zhan QQ, Wang YL, He SL. Photosensitizer encapsulated organically modified silica nanoparticles for direct two-photon photodynamic therapy and In Vivo functional imaging. Biomaterials. 2012; 33: 4851-60.

10. Starkey JR, Rebane AK, Drobizhev MA, Meng FQ, Gong AJ, Elliott A, et al. New Two-Photon Activated Photodynamic Therapy Sensitizers Induce Xenograft Tumor Regressions after Near-IR Laser Treatment through the Body of the Host Mouse. Clin Cancer Res. 2008; 14: 6564-73.

11. Chen G, Shen J, Ohulchanskyy TY, Patel NJ, Kutikov A, Li Z, et al. (alpha-NaYbF(4): $\operatorname{Tm}(3+)) / \mathrm{CaF}(2) \quad$ Core/Shell Nanoparticles with Efficient Near-Infrared to Near-Infrared Upconversion for High-Contrast Deep Tissue Bioimaging. Acs Nano. 2012; 6: 8280-7.

12. Wang J, Wang F, Wang C, Liu Z, Liu XG. Single-Band Upconversion Emission in Lanthanide-Doped KMnF3 Nanocrystals. Angew Chem Int Edit. 2011; 50: 10369-72.

13. Zhou J, Liu Z, Li FY. Upconversion nanophosphors for small-animal imaging. Chem Soc Rev. 2012; 41: 1323-49..

14. Bouzigues C, Gacoin T, Alexandrou A. Biological applications of rare-earth based nanoparticles. Acs Nano. 2011; 5: 8488-505.
15. Shen J, Zhao L, Han G. Lanthanide-doped upconverting luminescent nanoparticle platforms for optical imaging-guided drug delivery and therapy. Adv Drug Deliv Rev. 2012; doi:10.1016/j.addr.2012.05.007.

16. Wang F, Banerjee D, Liu YS, Chen XY, Liu XG. Upconversion nanoparticles in biological labeling, imaging, and therapy. Analyst. 2010; 135: 1839-54.

17. Haase M, Schafer H. Upconverting Nanoparticles. Angew Chem Int Edit. 2011; 50: 5808-29.

18. Auzel F. Upconversion and anti-stokes processes with $\mathrm{f}$ and $\mathrm{d}$ ions in solids. Chem Rev. 2004; 104: 139-73.

19. Chatterjee DK, Gnanasammandhan MK, Zhang Y. Small upconverting fluorescent nanoparticles for biomedical applications. Small. 2010; 6: 2781-95.

20. Wang C, Cheng L, Liu Z. Upconversion nanoparticles for potential cancer theranostics. Therapeutic delivery. 2011; 2: 1235-9.

21. Li CX, Lin J. Rare earth fluoride nano-/microcrystals: synthesis, surface modification and application. J Mater Chem. 2010; 20: 6831-47.

22. Cheng L, Wang C, Liu Z. Upconversion Nanoparticles and Their Composite Nanostructures for Biomedical Imaging and Cancer Therapy. Nanoscale. 2012; DOI: 10.1039/C2NR32311G.

23. Cheng L, Wang $C$, Ma X, Wang $Q$, Cheng $Y$, Wang $H$, et al. Multifunctional Upconversion Nanoparticles for Dual-Modal Imaging-Guided Stem Cell Therapy under Remote Magnetic Control. Adv Funct Mater. 2012; doi:10.1002/adfm.201201733.

24. Idris NM, Li ZQ, Ye L, Sim EKW, Mahendran R, Ho PCL, et al. Tracking transplanted cells in live animal using upconversion fluorescent nanoparticles. Biomaterials. 2009; 30: 5104-13.

25. Wang $\mathrm{C}, \mathrm{Cheng} \mathrm{L}, \mathrm{Xu} \mathrm{H}$, Liu Z. Towards whole-body imaging at the single cell level using ultra-sensitive stem cell labeling with oligo-arginine modified upconversion nanoparticles. Biomaterials. 2012; 33: $4872-81$.

26. Liu Q, Sun Y, Yang TS, Feng W, Li CG, Li FY. Sub-10 nm Hexagonal Lanthanide-Doped NaLuF4 Upconversion Nanocrystals for Sensitive Bioimaging in Vivo. J Am Chem Soc. 2011; 133: 17122-5.

27. Cheng LA, Yang K, Zhang SA, Shao MW, Lee ST, Liu ZA. Highly-Sensitive Multiplexed in vivo Imaging Using PEGylated Upconversion Nanoparticles. Nano Res. 2010; 3: 722-32.

28. Li ZQ, Zhang Y, Jiang S. Multicolor Core/Shell-Structured Upconversion Fluorescent Nanoparticles. Adv Mater. 2008; 20: 4765-69

29. Cheng LA, Yang K, Shao MW, Lee ST, Liu ZA. Multicolor In Vivo Imaging of Upconversion Nanopaticles with Emissions Tuned by Luminescence Resonance Energy Transfer. J Phys Chem C. 2011; 115: 2686-92.

30. Ju Q, Tu DT, Liu YS, Li RF, Zhu HM, Chen JC, et al. Amine-Functionalized Lanthanide-Doped KGdF4 Nanocrystals as Potential Optical/Magnetic Multimodal BioProbes. J Am Chem Soc. 2012; 134: 1323-30.

31. Wang F, Han Y, Lim CS, Lu YH, Wang J, Xu J, et al. Simultaneous phase and size control of upconversion nanocrystals through lanthanide doping. Nature. 2010; 463: 1061-5.

32. Xue XJ, Wang F, Liu XG. Emerging functional nanomaterials for therapeutics. J Mater Chem. 2011; 21: 13107-27.

33. Mader HS, Kele P, Saleh SM, Wolfbeis OS. Upconverting luminescent nanoparticles for use in bioconjugation and bioimaging. Curr Opin Chem Biol. 2010; 14: 582-96.

34. Shen J, Zhao L, Han G. Lanthanide-doped upconverting luminescent nanoparticle platforms for optical imaging-guided drug delivery and therapy. Adv Drug Deliver Rev. 2012; doi:10.1016/j.addr.2012.05.007.

35. Xiong LQ, Chen ZG, Tian QW, Cao TY, Xu CJ, Li FY. High Contrast Upconversion Luminescence Targeted Imaging in Vivo Using Peptide-Labeled Nanophosphors. Anal Chem. 2009; 81: 8687-94.

36. Zhou J, Yu MX, Sun Y, Zhang XZ, Zhu XJ, Wu ZH, et al. Fluorine-18-labeled Gd3+/Yb3+/Er3+ co-doped NaYF4 nanophosphors for multimodality PET/MR/UCL imaging. Biomaterials. 2011; 32: 1148-56.

37. Zhang P, Steelant W, Kumar M, Scholfield M. Versatile photosensitizers for photodynamic therapy at infrared excitation. J Am Chem Soc. 2007; 129: 4526-27.

38. Lim ME, Lee YL, Zhang Y, Chu JJH. Photodynamic inactivation of viruses using upconversion nanoparticles. Biomaterials. 2012; 33: 1912-20.

39. Chen F, Zhang SI, Bu WB, Chen $Y$, Xiao QF, Liu JA, et al. A Uniform Sub-50 nm-Sized Magnetic/Upconversion Fluorescent Bimodal Imaging Agent Capable of Generating Singlet Oxygen by Using a $980 \mathrm{~nm}$ Laser. Chem-Eur J. 2012; 18: 7082-90. 
40. Zhao Z, Han Y, Lin C, Hu D, Wang F, Chen X, et al. Multifunctional core-shell upconverting nanoparticles for imaging and photodynamic therapy of liver cancer cells. Chemistry, an Asian journal. 2012; 7: 830-7.

41. Qiao XF, Zhou JC, Xiao JW, Wang YF, Sun LD, Yan CH. Triple-functional core-shell structured upconversion luminescent nanoparticles covalently grafted with photosensitizer for luminescent, magnetic resonance imaging and photodynamic therapy in vitro. Nanoscale. 2012; 4: 4611-23.

42. Chatterjee DK, Gnanasammandhan MK, Zhang Y. Small Upconverting Fluorescent Nanoparticles for Biomedical Applications. Small. 2010; 6: 2781-95.

43. Guo HC, Qian HS, Idris NM, Zhang Y. Singlet oxygen-induced apoptosis of cancer cells using upconversion fluorescent nanoparticles as a carrier of photosensitizer. Nanomed-Nanotechnol. 2010; 6: 486-95.

44. Shan JN, Budijono SJ, Hu GH, Yao N, Kang YB, Ju YG, et al. Pegylated Composite Nanoparticles Containing Upconverting Phosphors and meso-Tetraphenyl porphine (TPP) for Photodynamic Therapy. Adv Funct Mater. 2011; 21: 2488-95.

45. Ungun B, Prud'homme RK, Budijono SJ, Shan JN, Lim SF, Ju YG, et al. Nanofabricated upconversion nanoparticles for photodynamic therapy. Opt Express. 2009; 17: 80-6.

46. Qian HS, Guo HC, Ho PCL, Mahendran R, Zhang Y. Mesoporous-Silica-Coated Up-Conversion Fluorescent Nanoparticles for Photodynamic Therapy. Small. 2009; 5: 2285-90.

47. Wang C, Cheng LA, Liu ZA. Drug delivery with upconversion nanoparticles for multi-functional targeted cancer cell imaging and therapy. Biomaterials. 2011; 32: 1110-20.

48. Wang C, Tao HQ, Cheng L, Liu Z. Near-infrared light induced in vivo photodynamic therapy of cancer based on upconversion nanoparticles. Biomaterials. 2011; 32: 6145-54.

49. Liu K, Liu X, Zeng Q, Zhang Y, Tu L, Liu T, et al. Covalently assembled NIR nanoplatform for simultaneous fluorescence imaging and photodynamic therapy of cancer cells. Acs Nano. 2012; 6: 4054-62.

50. Zhou A, Wei Y, Wu B, Chen Q, Xing D. Pyropheophorbide A and c(RGDyK) comodified chitosan-wrapped upconversion nanoparticle for targeted near-infrared photodynamic therapy. Mol Pharm. 2012; 9: 1580-9.

51. Park YI, Kim HM, Kim JH, Moon KC, Yoo B, Lee KT, et al. Theranostic Probe Based on Lanthanide-Doped Nanoparticles for Simultaneous In Vivo Dual-Modal Imaging and Photodynamic Therapy. Adv Mater. 2012; doi:10.1002/adma.201202433.

52. Chatterjee DK, Yong Z. Upconverting nanoparticles as nanotransducers for photodynamic therapy in cancer cells. Nanomedicine-Uk. 2008; 3: 73-82.

53. Cui SS, Chen HY, Zhu HY, Tian JM, Chi XM, Qian ZY, et al. Amphiphilic chitosan modified upconversion nanoparticles for in vivo photodynamic therapy induced by near-infrared light. J Mater Chem. 2012; 22: 4861-73.

54. Jayakumar MKG, Idris NM, Zhang Y. Remote activation of biomolecules in deep tissues using near-infrared-to-UV upconversion nanotransducers. P Natl Acad Sci USA. 2012; 109: 8483-8.

55. Carling CJ, Nourmohammadian F, Boyer JC, Branda NR. Remote-Control Photorelease of Caged Compounds Using Near-Infrared Light and Upconverting Nanoparticles. Angew Chem Int Edit. 2010; 49: 3782-5.

56. Yan B, Boyer JC, Branda NR, Zhao Y. Near-Infrared Light-Triggered Dissociation of Block Copolymer Micelles Using Upconverting Nanoparticles. J Am Chem Soc. 2011; 133: 19714-7.

57. Liu R, Tu DT, Liu YS, Zhu HM, Li RF, Zheng W, et al. Controlled synthesis and optical spectroscopy of lanthanide-doped KLaF4 nanocrystals. Nanoscale. 2012; 4: 4485-91.

58. Qiu Y, Park K. Environment-sensitive hydrogels for drug delivery. Adv Drug Deliv Rev. 2001; 53: 321-39.

59. Tian G, Gu ZJ, Zhou LJ, Yin WY, Liu XX, Yan L, et al. Mn2+ Dopant-Controlled Synthesis of NaYF4:Yb/Er Upconversion Nanoparticles for in vivo Imaging and Drug Delivery. Adv Mater. 2012; 24: 1226-31.

60. Dai YL, Ma PA, Cheng ZY, Kang XJ, Zhang X, Hou ZY, et al. Up-Conversion Cell Imaging and pH-Induced Thermally Controlled Drug Release from NaYF4:Yb3+/Er3+@Hydrogel Core-Shell Hybrid Microspheres. Acs Nano. 2012; 6: 3327-38.

61. Hou ZY, Li CX, Ma PA, Cheng ZY, Li XJ, Zhang X, et al. Up-Conversion Luminescent and Porous NaYF4:Yb3+, Er3+@SiO2 Nanocomposite Fibers for Anti-Cancer Drug Delivery and Cell Imaging. Adv Funct Mater. 2012; 22: 2713-22.

62. Cheng L, Yang K, Li Y, Chen J, Wang C, Shao M, et al. Facile preparation of multifunctional upconversion nanoprobes for multimodal imaging and dual-targeted photothermal therapy. Angew Chem Int Ed Engl. 2011; 50: 7385-90.
63. Cheng L, Yang K, Li Y, Zeng X, Shao M, Lee ST, et al. Multifunctional nanoparticles for upconversion luminescence/MR multimodal imaging and magnetically targeted photothermal therapy. Biomaterials. 2012; 33: 2215-22.

64. Dai Y, Yang D, Ma Pa, Kang X, Zhang X, Li C, et al. Doxorubicin conjugated NaYF4:Yb3+/Tm3+ nanoparticles for therapy and sensing of drug delivery by luminescence resonance energy transfer. Biomaterials. 2012; 33: 8704-13.

65. Xu H, Cheng L, Wang C, Ma XX, Li YG, Liu Z. Polymer encapsulated upconversion nanoparticle/iron oxide nanocomposites for multimodal imaging and magnetic targeted drug delivery. Biomaterials. 2011; 32: 9364-73.

66. Gai SL, Yang PP, Li CX, Wang WX, Dai YL, Niu N, et al. Synthesis of Magnetic, Up-Conversion Luminescent, and Mesoporous Core-Shell-Structured Nanocomposites as Drug Carriers. Adv Funct Mater. 2010; 20: 1166-72.

67. Zhang F, Braun GB, Pallaoro A, Zhang YC, Shi YF, Cui DX, et al. Mesoporous Multifunctional Upconversion Luminescent and Magnetic "Nanorattle" Materials for Targeted Chemotherapy. Nano Lett. 2012; 12: 61-7.

68. Hou ZY, Li CX, Ma PA, Li GG, Cheng ZY, Peng C, et al. Electrospinning Preparation and Drug-Delivery Properties of an Up-conversion Luminescent Porous NaYF4:Yb3+, Er3+@Silica Fiber Nanocomposite. Adv Funct Mater. 2011; 21: 2356-65.

69. Xu ZH, Ma PA, Li CX, Hou ZY, Zhai XF, Huang SS, et al. Monodisperse core-shell structured up-conversion $\mathrm{Yb}(\mathrm{OH}) \mathrm{CO} 3 @ Y b P O 4: E r 3+$ hollow spheres as drug carriers. Biomaterials. 2011; 32: 4161-73.

70. Boyer JC, van Veggel FCJM. Absolute quantum yield measurements of colloidal NaYF4: Er3+, Yb3+ upconverting nanoparticles. Nanoscale. 2010; 2: 1417-9.

71. Wang F, Deng RR, Wang J, Wang QX, Han Y, Zhu HM, et al. Tuning upconversion through energy migration in core-shell nanoparticles. Nature Materials. 2011; 10: 968-73.

72. Zhou J, Zhu XJ, Chen M, Sun Y, Li FY. Water-stable NaLuF4-based upconversion nanophosphors with long-term validity for multimodal lymphatic imaging. Biomaterials. 2012; 33: 6201-10.

73. Zhan QQ, Qian J, Liang HJ, Somesfalean G, Wang D, He SL, et al. Using 915 nm Laser Excited Tm3+/Er3+/Ho3+-Doped NaYbF4 Upconversion Nanoparticles for in Vitro and Deeper in Vivo Bioimaging without Overheating Irradiation. Acs Nano. 2011; 5: 3744-57.

74. Jalil RA, Zhang Y. Biocompatibility of silica coated NaYF4 upconversion fluorescent nanocrystals. Biomaterials. 2008; 29: 4122-8.

75. Xiong LQ, Yang TS, Yang Y, Xu CJ, Li FY. Long-term in vivo biodistribution imaging and toxicity of polyacrylic acid-coated upconversion nanophosphors. Biomaterials. 2010; 31: 7078-85.

76. Cheng L, Yang $\mathrm{K}$, Shao MW, Lu XH, Liu Z. In vivo pharmacokinetics, long-term biodistribution and toxicology study of functionalized upconversion nanoparticles in mice. Nanomedicine-Uk. 2011; 6: 1327-40. 